\title{
Black rice-derived anthocyanins inhibit HER-2-positive breast cancer epithelial-mesenchymal transition-mediated metastasis in vitro by suppressing FAK signaling
}

\author{
JIE ZHOU*, YAN-FENG ZHU*, XIANG-YAN CHEN, BIN HAN, FEI LI, JING-YAO CHEN, \\ XIAO-LI PENG, LI-PING LUO, WEI CHEN and XIAO-PING YU \\ Department of Public Health, Chengdu Medical College, Chengdu, Sichuan 610500, P.R. China
}

Received March 8, 2016; Accepted September 13, 2017

DOI: $10.3892 / \mathrm{ijmm} .2017 .3183$

\begin{abstract}
This study aimed to investigate the role of focal adhesion kinase (FAK) signaling in the inhibitory effects of black rice anthocyanins (BRACs) on human epidermal growth factor receptor-2 (HER-2)-positive human breast cancer cell metastasis, using the MCF-10A, MCF-7 and MDA-MB-453 cells. BRACs exerted an anti-metastatic effect on the HER-2-positive breast cancer cells. The effects of BRACs on the proliferation of the MDA-MB-453 cells were examined by cell counting kit-8 assay. A wound-healing assay was used to examine the effects of BRACs on the migration of the breast cancer cells. BRACs interrupted migration and invasion. BRACs decreased the migration distance of the HER-2-positive human breast cancer cells, MDA-MB-453, by $37 \%$ compared with the cells in the untreated group. They also reduced the number of invading MDA-MB-453 cells by $68 \%$. In addition, BRACs exerted an inhibitory effect on epithelial-mesenchymal transition. Western blot analysis revealed that BRACs decreased the phosphorylation of FAK, cSrc and p130 Cas. The FAK inhibitor, Y15, was also used to further evaluate the role of FAK signaling in the anti-metastatic effects of BRACs on MDA-MB- 453 cells. The results of western blot analysis revealed that BRACs increased the expression of the epithelial marker, E-cadherin, and decreased the expression of the mesenchymal markers, fibronectin and vimentin, in the MDA-MB-453 cells. In addition, BRACs decreased the interaction between HER-2 and FAK, FAK and $\mathrm{cSrc}, \mathrm{cSrc}$ and $\mathrm{p} 130^{\mathrm{Cas}}$, and between FAK and $\mathrm{p} 130^{\mathrm{Cas}}$. These results suggest that BRACs suppress the metastasis of HER-2-positive breast cancer in vitro, and that the cSrc/FAK/ p130 ${ }^{\text {Cas }}$ pathway plays a vital role in this inhibitory effect.
\end{abstract}

Correspondence to: Dr Xiao-Ping Yu, Department of Public Health, Chengdu Medical College, 783 Xindu Avenue, Xindu, Chengdu, Sichuan 610500, P.R. China

E-mail: cyggwsyxp@sina.com

\section{*Co-first authors}

Key words: black rice anthocyanins, human epidermal growth factor receptor-2, anti-metastasis, epithelial-mesenchymal transition, focal adhesion kinase

\section{Introduction}

Breast cancer is the most prevalent type of cancer with the highest incidence of all cancer types among women in China, accounting for $23 \%$ of diagnosed cancer cases and for $14 \%$ of cancer mortalities each year (1-3). Despite advances being made in the screening and adjuvant treatment of breast cancer, the mortality rates associated breast cancer remain high and metastasis is the leading cause of mortality (4). Human epidermal growth factor receptor-2 (HER-2) is a member of the epidermal growth factor receptor membrane tyrosine kinase, which plays an important role in the proliferation, invasion and migration of cells (5). An increased expression of HER-2 is found in $20-30 \%$ of patients with breast cancer and is associated with a poor prognosis and metastasis (6).

Tumor metastasis, an aggressive process in the development of tumor cells, is a multiple complex process involving invasion and migration. Epithelial-mesenchymal transition (EMT), an important process originally described in embryonic development, has been reported to play a vital role in the metastasis of some carcinomas (7). During the process of EMT, focal adhesion kinase (FAK) promotes the phosphorylation of $\beta$-catenin to increase the expression of transcription factors associated with EMT.

FAK is a non-receptor tyrosine kinase that has been shown to be upregulated in various human cancer cells, including lung, colon, thyroid, hepatocellular, prostate, pancreatic and breast cancers ( 8 and refs therein). It has also been reported that FAK plays a vital role in various biological processes, including adhesion, angiogenesis, motility, EMT and metastasis (9). It has also been reported that HER-2 influences the metastasis of breast cancer cells through the cSrc/FAK pathway (10). The activation of cSrc/FAK is critical for the phosphorylation of $\mathrm{p} 130^{\mathrm{Cas}}$. The interaction of $\mathrm{p} 130^{\mathrm{Cas}}$ with FAK promotes cell migration by activating c-Jun N-terminal kinases (JNKs) (11).

Recently, researches have studied phytochemicals extracted from fruits, vegetables and other herbs. It has been defined that these nutrients, including anthocyanins, play an important role on oncotherapy (12). A previous study suggested that a phytochemical named oleuropein reduced tumor growth in xenografted mice and enhanced the efficacy of trastuzumab against breast cancer cells (13). Citrus fruits and their bioactive 
ingredients have also been shown to suppress cancer growth by inducing apoptsis (14). The overexpression of oncogenes, the inactivation of tumor-related suppressor genes and the loss of apoptosis are some of the mechanisms that have been widely researched in molecular oncology. Phytochemicals, such as fungal substances has been shown to control the expression of oncogenes and tumor suppressor in vivo and in vitro (15 and refs therein). Rutin modulates intracellular signaling pathways to control cancer cell growth and apoptosis in vivo and in vitro (16 and refs therein). Viscum album has also emerged as a natural source with substantial biological activities, such as modulating endoplasmic reticulum stress and targeting cytoskeletal machinery in cancer cells (17).

Black rice anthocyanins (BRACs) are a category of anthocyanins extracted from the aleurone layer of black rice, which is regarded as a healthy food due to its beneficial effects on the liver and gastrointestinal tract (18). It has been demonstrated that anthocyanins exert an inhibitory effect on carcinogenesis, and inhibit cancer progression and metastasis through cell signal transduction (19). Hui et al (20) reported that BRACs induced the apoptosis and inhibited the angiogenesis of MDA-MB-453 cells through an intrinsic pathway. In their study, BALB/c nude mice bearing MDA-MB-453 tumor xenografts orally ingested BRACs (100 mg/kg/day), which suppressed tumor growth and angiogenesis by decreasing the expression of angiogenic factors. Previous studies have also indicated that BRACs inhibited lung nodule formation and pulmonary metastasis in ErbB2-positive MDA-MB-453 subcutaneous xenografts, and that the RAS/RAF/MAPK pathway played a vital role in this inhibitory effect $(21,22)$. However, limited information is available on the inhibitory effects of BRACs on EMT and FAK signaling associated with this anti-metastatic effect.

The present study thus investigated whether FAK signaling plays a role in the anti-metastatic effects of BRACs on HER-2positive breast cancer in vitro. It was found that BRACs exert a suppressive effect on the process of EMT.

\section{Materials and methods}

Reagents. The BRACs used in this study were purchased from the New Star Natural Plant Development Company (Jilin, China). The FAK inhibitor, 1,2,4,5-benzenetetramine tetrahydrochloride (Y15), was obtained from Sigma (St. Louis, MO, USA). Fetal bovine serum (FBS) and horse serum were purchased from Gibco-BRL (Grand Island, NY, USA). Dulbecco's modified Eagle's medium (DMEM)/high glucose and DMEM/F12 were purchased from HyClone (Beijing, China). L15 medium was obtained from Keygen Biotech (Nanjing, China). Primary antibodies against FAK (ab40794), phospho-FAK (ab38458, ab81298), Src (ab16885), phospho-Src (ab24789), and p130 ${ }^{\mathrm{Cas}}$ (ab136514) were purchased from Abcam (Cambridge, MA, USA). Phospho-p130 Cas (\#4011) antibody was purchased from Cell Signaling Technology (Danvers, MA, USA). Primary antibodies against E-cadherin (610181), vimentin (550513), and fibronectin (610077) were purchased from BD Biosciences (Bedford, MA, USA).

Cell culture. The human breast cancer cell lines, MCF-7 (HER-2 negative), MDA-MB-453 (HER-2 positive), and the normal breast cell line, MCF-10A, were purchased from the Chinese Academy of Science. The MCF-7 cells were cultured in DMEM/ high glucose with $10 \%$ FBS, and the MCF-10A cells were cultured in DMEM/F12 supplemented with $6 \%$ horse serum, $10 \mu \mathrm{g} / \mathrm{ml}$ insulin (Wako, Osaka, Japan), $20 \mathrm{ng} / \mathrm{ml}$ epidermal growth factor (PeproTech, Rocky Hill, NJ, USA), 100 ng/ml cholera toxin and $0.5 \mu \mathrm{g} / \mathrm{ml}$ hydrocortisone in humidified $5 \% \mathrm{CO}_{2}$ at $37^{\circ} \mathrm{C}$. The MDA-MB-453 cells were cultured in L15 medium with $10 \% \mathrm{FBS}$ in $100 \% \mathrm{O}_{2}$ at $37^{\circ} \mathrm{C}$.

Cell counting kit-8 (CCK-8) assay. The MCF-10A, MCF-7 and MDA-MB-453 cells ( $1 \times 10^{3}$ cells/well in a 96-well plate) were incubated overnight at $4^{\circ} \mathrm{C}$. Following incubation, the cells were treated with or without BRACs at various concentrations $(0,50,100,200$ and $400 \mu \mathrm{g} / \mathrm{ml})$ for $24 \mathrm{~h}$. CCK-8 assays (C0037; Beyotime, Shanghai, China) were performed according to the instructions provided with the kits, and the absorbance was detected at $450 \mathrm{~nm}$ using a microplate reader (Biotek, Shanghai, China).

Cell adhesion assay. The cell adhesion assay was performed as described in a previous study (23). Briefly, the cells were pre-treated with various concentrations of BRACs $(0,50,100$, 200 and $400 \mu \mathrm{g} / \mathrm{ml})$ or Y15 $(0,2,4,8$ and $16 \mu \mathrm{M})$ for $24 \mathrm{~h}$. A 96-well plate was pre-coated with fibronectin $(10 \mu \mathrm{g} / \mathrm{ml})$ for $1 \mathrm{~h}$ at $37^{\circ} \mathrm{C}$. The plate was then incubated with $3 \%$ bovine serum albumin (BSA) in phosphate-buffered saline (PBS) for $30 \mathrm{~min}$ to block non-specific binding sites; the cells $\left(5 \times 10^{4} /\right.$ well) were then seeded on a fibronectin pre-coated plate at $37^{\circ} \mathrm{C}$ with $5 \% \mathrm{CO}_{2}$, and the plate was then washed with PBS 3 times to remove the unattached cells after $1 \mathrm{~h}$. The attached cells were fixed with $3.7 \%$ paraformaldehyde for $10 \mathrm{~min}$ and stained with $0.1 \%$ crystal violet (C0121, Beyotime) in $2 \%$ ethanol for $10 \mathrm{~min}$ followed by washing 3 times with PBS. The plate with attached the cells was rinsed with water and dried. The cells with crystal violet were eluted in $10 \%$ acetic acid, and the absorbance was measured at $595 \mathrm{~nm}$ using a microplate reader (Thermo Fisher Scientific, Inc., Waltham, MA, USA).

Wound healing assay. The MCF-10A, MCF-7, and MDA-MB-453 cells were grown in 24-well plates to form a confluent monolayer. A cross-scratch wound was created using 200- $\mu 1$ sterile pipette tips. The cells were then treated with BRACs $(200 \mu \mathrm{g} / \mathrm{ml})$ or Y15 $(10 \mu \mathrm{M})$ for $24 \mathrm{~h}$. The images were observed under an inverted light microscope (Olympus, Tokyo, Japan). Migration distances were measured using ImageJ software.

Cell invasion assay. The in vitro invasion features of the cells were measured by a cell invasion assay (ECM550; Millipore, Billerica, MA, USA), according to the manufacturer's instructions. The cells ( $3 \times 10^{5} /$ chamber) were seeded in the ECM-coated upper compartment, and media including BRACs $(200 \mu \mathrm{g} / \mathrm{ml})$ or Y15 $(10 \mu \mathrm{M})$ were then added to each upper insert. The lower compartment was filled with a medium containing serum. Following $48 \mathrm{~h}$ of incubation at $37^{\circ} \mathrm{C}$, non-invading cells were removed using cotton swabs, and the Transwell membrane was then fixed and stained with crystal violet. The number of invading cells was counted in random fields under a light microscope (DM4000B; Leica, Wetzlar, Germany) followed by several times rinses in water. 

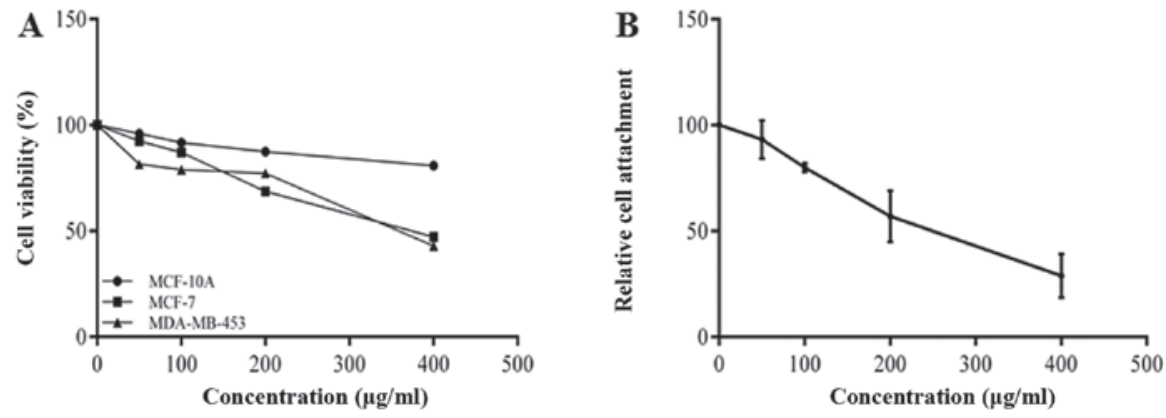

Figure 1. Inhibitory effects of black rice anthocyanins (BRACs) on cell adhesion and viability. (A) Cell counting kit-8 (CCK-8) assays were performed on cells treated with various concentrations of BRACs. The average cell viability as a percentage is shown. Data are the means \pm SD of 4 replicated experiments. (B) MDA-MB-453 cells were treated with various concentrations of BRACs for $24 \mathrm{~h}$, and the cells in each group were then incubated on a $96-$ well cell culture plate pre-coated with fibronectin. Relative cell attachment values are shown. Data are the means \pm SD of 4 replicated experiments.

Western blot analysis. The cells pre-treated with BRACs $(200 \mu \mathrm{g} /$ $\mathrm{ml})$ or $\mathrm{Y} 15(10 \mu \mathrm{M})$ were lysed in a radioimmunoprecipitation assay (RIPA) buffer [50 mM Tris-HCL (pH 7.4), $150 \mathrm{mM} \mathrm{NaCl}$, $1 \%$ Triton $\mathrm{X}-100,1 \%$ sodium deoxycholate, $0.1 \%$ sodium dodecyl sulfate (SDS) and $1 \mathrm{mM}$ phenylmethanesulfonyl fluoride] supplemented with protease inhibitor cocktail tablets for $30 \mathrm{~min}$ at $4^{\circ} \mathrm{C}$. Lysates were centrifuged at $13,000 \mathrm{rpm}$ for $30 \mathrm{~min}$ at $4^{\circ} \mathrm{C}$, and the undissolved debris was discarded. The protein concentration was detected using biocinchoninic acid assay kits (P0010; Beyotime). Equal amounts of protein $(40 \mu \mathrm{g})$ were separated on sodium dodecyl sulfate-polyacrylamide gel electrophoresis and transferred onto polyvinylidene fluoride membranes (Millipore). The membranes were blocked with TBST $[20 \mathrm{mM}$ Tris-HCL ( $\mathrm{pH}$ 7.4), $150 \mathrm{mM} \mathrm{NaCl}, 0.1 \%$ Tween-20] containing 5\% non-fat milk or $3 \%$ BSA. After blocking, the membranes were incubated with primary antibodies $(1: 1,000)$ overnight at $4^{\circ} \mathrm{C}$. The membranes were washed 3 times with TBST followed by incubation with horseradish peroxidase-conjugated secondary antibodies (1:10,000; Beyotime) for $1 \mathrm{~h}$ at room temperature. The proteins were detected using an enhanced chemiluminescence detection kit (Thermo Fisher Scientific, Inc.).

Immunoprecipitation analysis. The cells were rinsed twice with cold PBS, followed by lysing with RIPA buffer containing phosphatase inhibitors. Anti-ErbB2, FAK, cSrc, or p130 Cas antibodies were added to protein $\mathrm{A} / \mathrm{G}$ agarose beads (Millipore) and incubated for $1 \mathrm{~h}$ at $37^{\circ} \mathrm{C}$ with gentle mixing. The mixture was centrifuged at $4^{\circ} \mathrm{C}$, the supernatant was discarded, and the beads were washed 3 times with PBST. Equal amounts of proteins were added to the beads for incubation overnight at $4^{\circ} \mathrm{C}$ with gentle shaking. The beads were suspended by boiling with sample buffer after washing with PBST and analyzed by western blot analysis.

Statistical analysis. Data are presented as the means \pm standard deviation (SD). One-way analysis of variance and Dunnett's t-test were used to determine the statistical significance between values of the various experimental and control groups. A value of $\mathrm{P}<0.05$ was considered to indicate a statistically significant difference.

\section{Results}

BRACs inhibit the metastasis of Her-2-positive breast cancer cells. The effects of BRACs on the proliferation of
MDA-MB-453 cells were examined by CCK-8 assay. The results shown in Fig. 1A indicate that BRACs inhibited the viability of the MDA-MB-453 cells in a dose-dependent manner. A wound healing assay was also used to examine the effect of BRACs on the migration of the breast cancer cells. BRACs significantly decreased the migration distance of MDA-MB-453 cells by $37 \%$ compared with the untreated cells after $24 \mathrm{~h}$ (Fig. 2A and $\mathrm{C}$ ). The results also revealed that BRACs did not affect the migration ability of the normal mammary epithelial MCF-10A cells. In addition, a Matrigel-coated membrane was used to examine the invasive ability of the MDA-MB-453 cells. The results indicated that BRACs decreased the invasive ability of the MDA-MB-453 cells, the number of invaded cells has been reduced by $68 \%$ (Fig. $2 \mathrm{~B}$ and $\mathrm{D}$ ). The adhesion of the cancer cells to extracellular matrix (ECM) and cell-ECM interaction are important processes of metastasis; therefore, this study examined the adhesion of the MDA-MB-453 cells to fibronectin, which is an essential protein in the ECM (24). BRACs inhibited the adhesion of the MDA-MB-453 cells to fibronectin in a concentration-dependent manner (Fig. 1B). These results suggest that BRACs exerts anti-metastatic effects on HER-2-positive breast cancer cells.

Y15, a small molecule inhibitor that inhibits the growth of breast cancers, can inhibit the phosphorylation of FAK by binding with the Y397 site. The Y397 site is an autophosphorylation site of FAK that provides a binding for $\mathrm{cSrc}$ to lead to downstream signaling by mediating the phosphorylation of FAK. Y15 significantly decreased the migration distance and the invasive ability of the MDA-MB-453 cells (Fig. 3). The adhesion between fibronectin and MDA-MB-453 cells was also reduced by Y15. Thus, Y15 exerted anti-metastatic effects similar to those of BRACs.

BRACs reduces EMT in Her-2-positive breast cancer cells. EMT plays an important role in the metastasis of breast carcinomas (7). The present study examined whether BRACs influence EMT in MDA-MB-453 cells. The morphology of the MDA-MB-453 cells significantly changed from a fibroblastlike to a cobblestone-like appearance following treatment with BRACs or Y15, suggesting that BRACs reduced EMT in the MDA-MB-453 cells (Fig. 4A). The results of western blot analysis also revealed that BRACs increased the expression of the epithelial marker, E-cadherin, and decreased the expression of the mesenchymal markers, fibronectin and vimentin, 


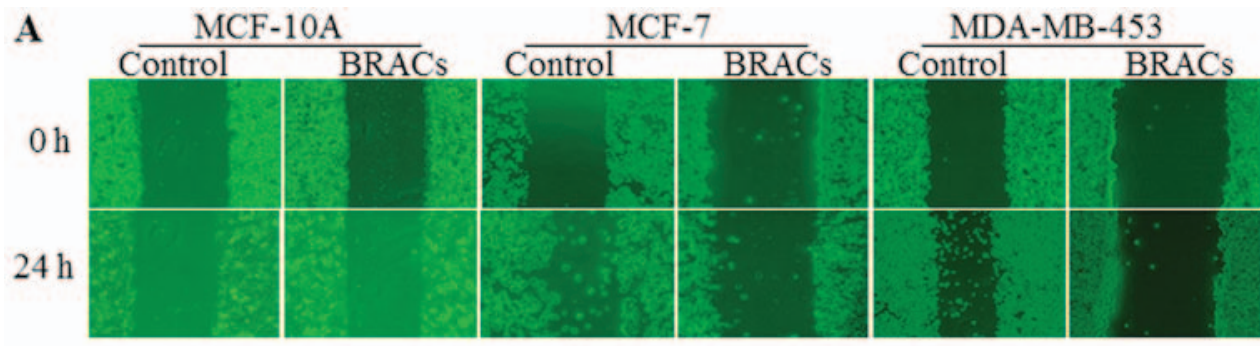

B

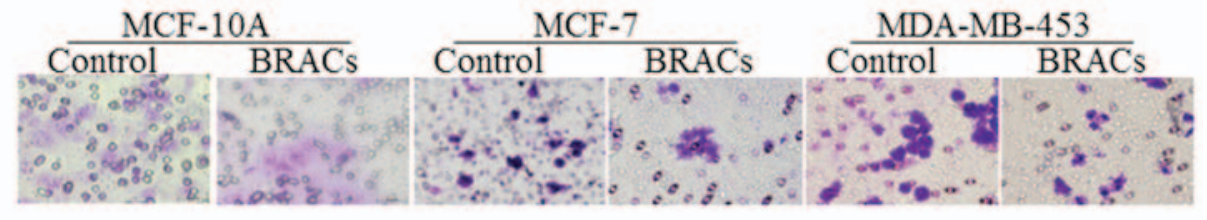

C

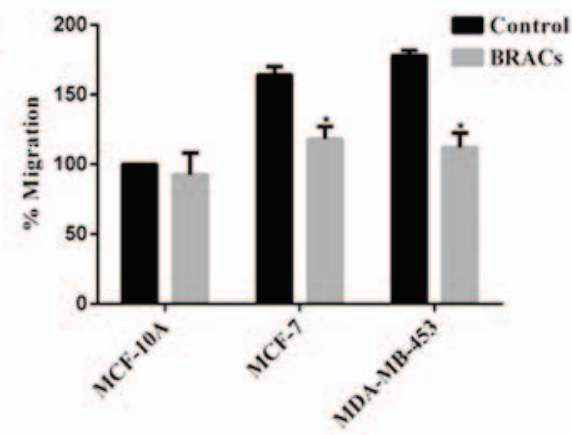

D

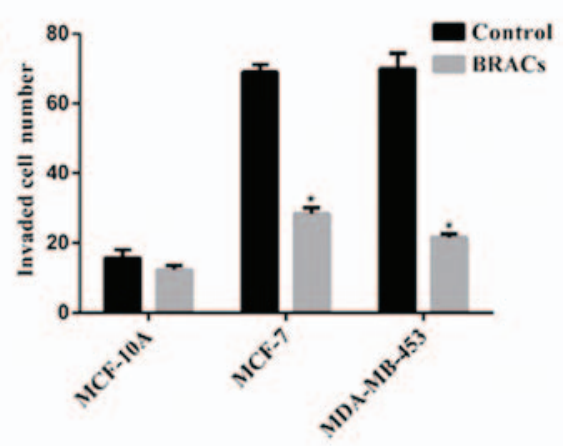

Figure 2. Inhibitory effects of black rice anthocyanins (BRACs) on cell migration and invasion. (A) The migration of cells was analyzed using a wound healing assay. Cells were observed under a microscope following treatment with or without BRACs ( 0 or $200 \mu \mathrm{g} / \mathrm{ml})$. (B) The invasion of cells was assessed by an $8-\mu \mathrm{m}$ pore size cell culture insert. Cells were treated with or without BRACs ( 0 or $200 \mu \mathrm{g} / \mathrm{ml})$ for $24 \mathrm{~h}$, followed by counting of the invading cells in 5 different visual fields counted from 4 independent experiments. (C) Migration distances were measured using ImageJ software. Values are presented as the means \pm SD of 4 replicated experiments. "P<0.05 denotes a significant difference from the control. (D) Invading cells are shown as the means \pm SD of 4 replicated experiments. ${ }^{*} \mathrm{P}<0.05$ denotes a significant difference from the control.

in the MDA-MB-453 cells (Fig. 4B). BRACs exerted an antiEMT effect that was similar to the effect of Y15 (Fig. 4B). BRACs together with Y15 further augmented the expression of E-cadherin and exerted further suppressive eftects on the expression of fibronectin and vimentin.

BRACs reduce EMT by inhibiting cSrc/FAK/p130 Cas signaling. $\mathrm{cSrc} / \mathrm{FAK} / \mathrm{p} 130^{\mathrm{Cas}}$ signaling plays a key role in favoring migration, invasion and metastasis in cancer cells regulated by EMT (10). To elucidate the molecular mechanisms underlying the anti-metastatic effects of BRACs, the effects of BRACs treatment on the expression and phosphorylation of cSrc/FAK/ p130 ${ }^{\text {Cas }}$ were evaluated by western blot analysis (Fig. 5A). BRACs significantly decreased the phosphorylation of cSrc, FAK and $130^{\mathrm{Cas}}$ in the MDA-MB-453 cells. Additional experiments were performed to determine the effects of BRACs on the association between HER-2 and FAK, FAK and cSrc, cSrc and $\mathrm{p} 130^{\mathrm{Cas}}$, and between FAK and $\mathrm{p} 130^{\mathrm{Cas}}$ by western blot analysis. BRACs treatment inhibited the interactions among these proteins (Fig. 5B-D). BRACs weakened the association between HER-2 and FAK, attenuated the activation of FAK by HER-2, and inhibited the contact among FAK, cSrc and p130 Cas proteins. It also reduced the interaction among these proteins further by suppressing the transduction of FAK signaling.
The results of western blot analysis revealed that Y15 also suppressed the phosphorylation of cSrc, FAK and $\mathrm{p} 130^{\mathrm{Cas}}$ in the MDA-MB-453 cells (Fig. 5E).

\section{Discussion}

Anthocyanins, a species of natural flavonoids existing widely in food such as cabbages, berries, grapes, cereal and other dark-colored fruits and vegetables, appear to have potential benefits such as antioxidant, anti-inflammatory, anticancerous and anti-metastatic activities (25). Anthocyanins exert their effects in a native or metabolized form by entering the circulation in a sufficient quantity. However, these beneficial effects are dependent on the bioavailability and absorbed substances of anthocyanins (26). As hydrolyzed by the colon microflora or intestinal enzymes, anthocyanins are usually absorbed slowly in the intestine due to the eliminated hydrophilic moiety which is usually necessary for anthocyanins to cross the intestine membrane via passive diffusion (27). Anthocyanins are then further metabolized in the liver and small intestine followed by entering the circulation and targeting different tissues in a conjugated structure by sulfation, glucuronidation or methylation. Ultimately, anthocyanins are secreted in bile or eliminated in urine (28). In biotransprotation, anthocyanins 


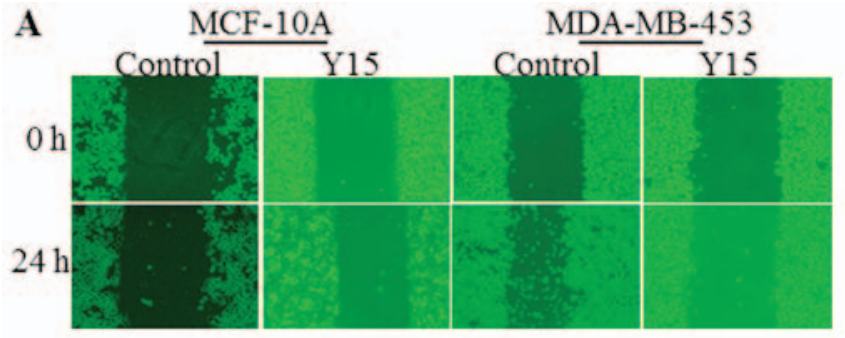

B

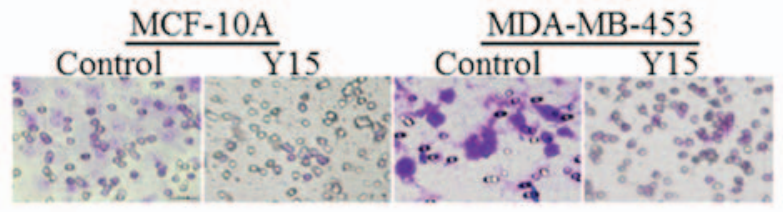

$\mathbf{E}$

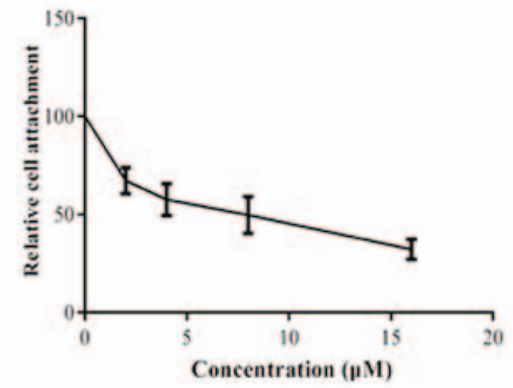

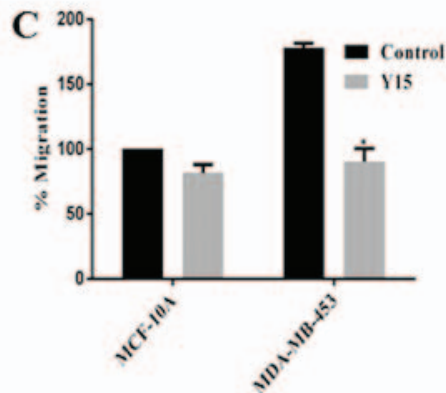

D

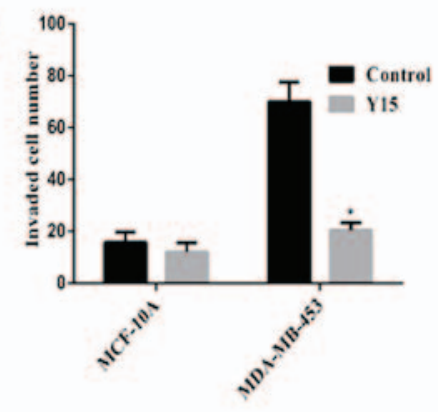

Figure 3. Inhibitory effects of Y15 on cell migration, invasion and adhesion. (A) The migration of cells was analyzed using a wound healing assay. (B) The invasion of cells was assessed by an $8-\mu \mathrm{m}$ pore size cell culture insert; invaded cells in 5 different visual fields were counted from 4 independent experiments. (C) Migration distances were measured using ImageJ software. Values are presented as the means \pm SD of 4 replicated experiments. "P $<0.05$ denotes a significant difference from the control. (D) Invading cells are shown as the means \pm SD for 4 replicated experiments. "P<0.05 denotes a significant difference from the control. (E) MDA-MB-453 cells were treated with various concentrations of Y15 for $24 \mathrm{~h}$, and the cells in each group were then incubated on a 96-well cell culture plate pre-coated with fibronectin. Relative cell attachment values are shown. Data are the means \pm SD of 4 replicated experiments.

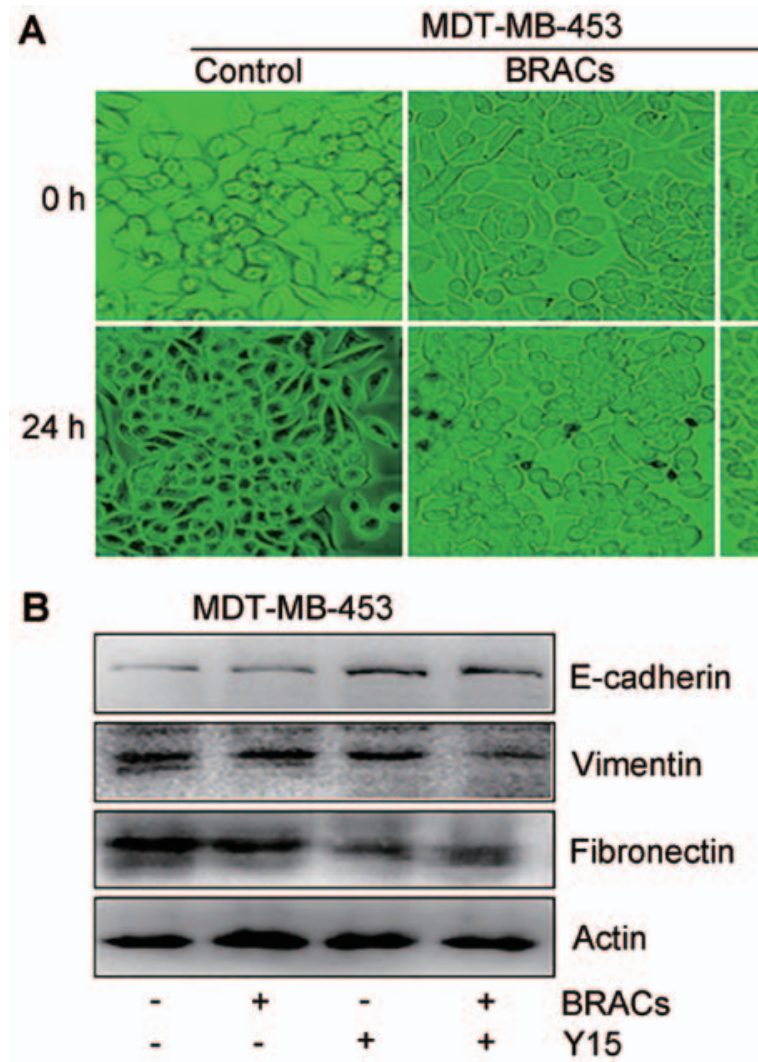

Figure 4. Inhibitory effects of black rice anthocyanins (BRACs) on epithelial-mesenchymal transition (EMT) in MDA-MB-453 cells. MDA-MB-453 cells were treated with BRACs $(200 \mu \mathrm{g} / \mathrm{ml})$ or Y15 $(10 \mu \mathrm{M})$ for $24 \mathrm{~h}$. (A) Morphology of the cells were observed and photographed using a microscope, bright field magnification, $x 40$. (B) Cells were harvested for the detection of expression of E-cadherin, vimentin and fibronectin by western blot analysis. 


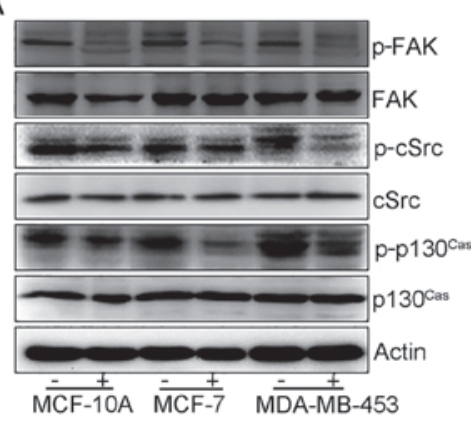

Treatment of cells with BRACs
B IP:HER-2

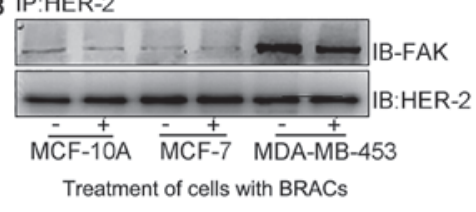

D IP:p130 cas

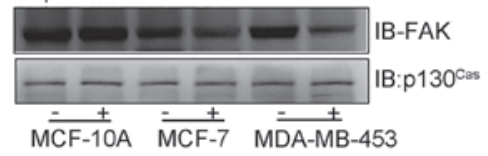

Treatment of cells with BRACs
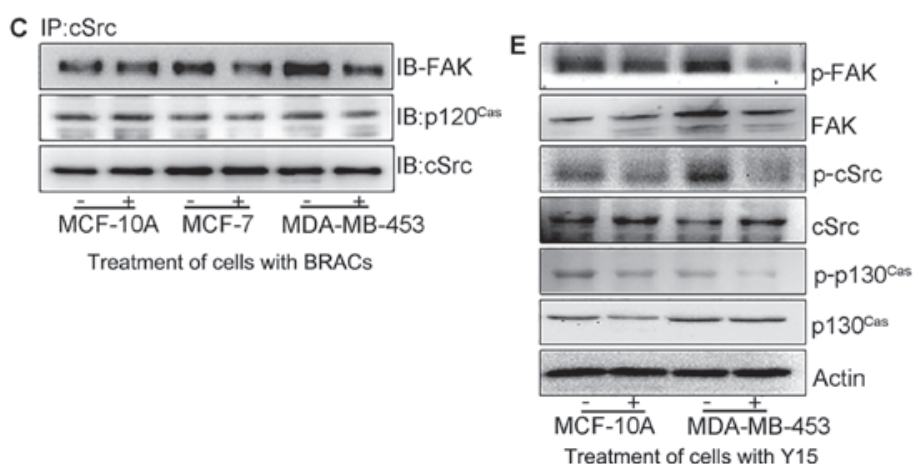

Figure 5. Effects of black rice anthocyanins (BRACs) or Y15 on the phosphorylation of focal adhesion kinase (FAK), cSrc, and p130 ${ }^{\mathrm{Cas}}$ and the interaction among these proteins. (A) Cells treated with/without BRACs were harvested for the detection of the phosphorylation of FAK, and cSrc, p130 Cas using western blot analysis. The expression of actin was used as a loading control. (B) Cells treated with/without BRACs were harvested for detecting the interaction between human epidermal growth factor receptor-2 (HER-2) and FAK by immunoprecipitation. The expression of HER-2 was used as a loading control. (C) The interaction among FAK, $\mathrm{CSrc}$ and $\mathrm{p} 130^{\mathrm{Cas}}$. The expression of $\mathrm{cSrc}$ was used as a loading control. (D) The interaction between FAK and p130 ${ }^{\mathrm{Cas}}$. The expression of p130 ${ }^{\mathrm{Cas}}$ was used as a loading control. (E) Cells treated with/without Y15 were harvested for detecting the phosphorylation of FAK, cSrc, and p130 Cas using western blot analysis. The expression of actin was used as a loading control.

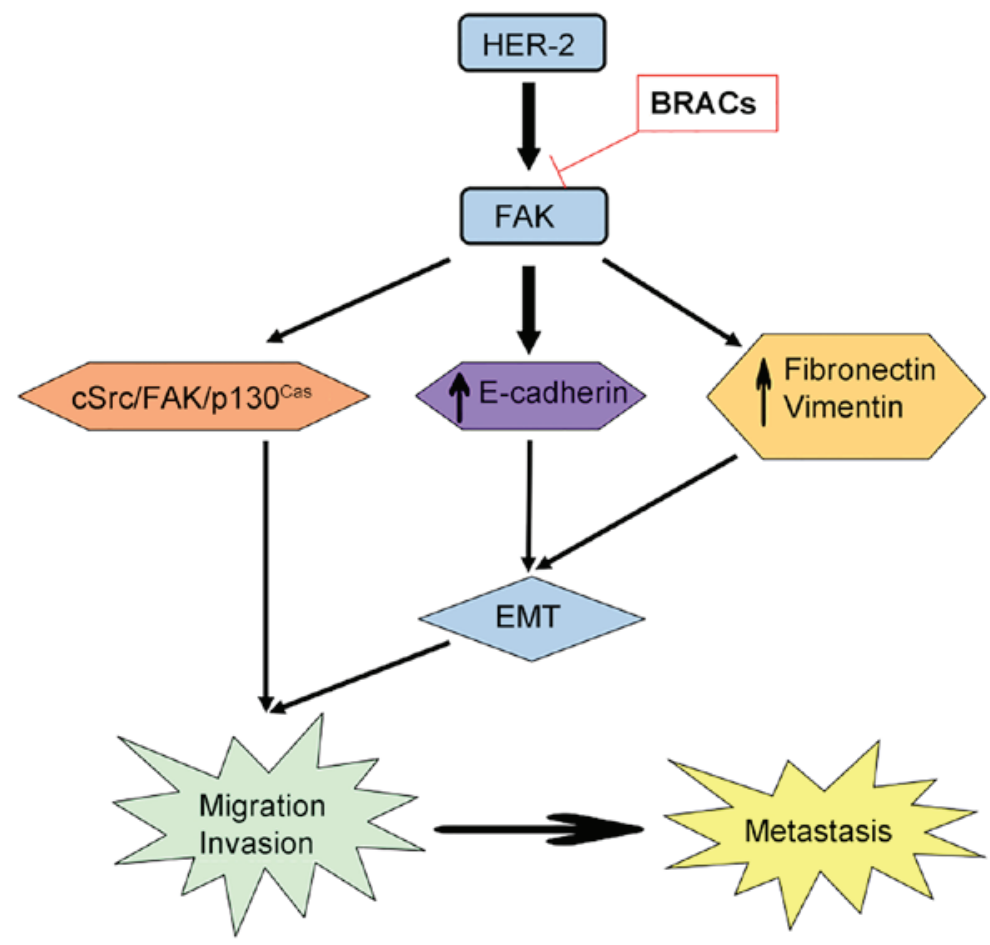

Figure 6. Proposed model for the role of focal adhesion kinase (FAK) in the metastasis of human epidermal growth factor receptor-2 (HER-2)-positive breast cancer cells. HER-2/FAK increases the phosphorylation of FAK/cSrc/p130 Cas signaling. The phosphorylation of FAK reduces the expression of the epithelial maker, E-cadherin, and increases the expression of the mesenchymal markers, vimentin and fibronectin, the leading processes of epithelial-mesenchymal transition (EMT). The aforementioned processes promote cell migration and invasion to facilitate the metastasisof breast cancer. 
can be transported through one human intestinal epithelial cell model in intact glycone forms (29), then stored in tissues rather than circulated in the blood circulation. Anthocyanins can be restored in the liver, cortex, eye and cerebellum after consuming blueberry anthocyanins, as shown in an in vivo study (30 and refs therein).

During the complex processes of metastasis, cancer cell invasion of the basement membrane is one of the earliest and critical steps (31). The most important step is the invasion of the ECM, known as the degradation of basement membrane collagen, by some proteolytic enzymes such as matrix metalloproteinases (MMP) and urokinase-type plasminogen activator (u-PA). BRACs have been found to inhibit cancer cell invasion by decreasing the expression of MMP and u-PA (32). The expression of MMP-2 and MMP-9 is regulated by FAK in the cell migration and invasion of hepatocellular carcinoma (33).

The present study indicated that BRACs reduced the adhesion, migration and invasion of human HER-2-positive breast cancer MDA-MB-453 cells. It also significantly altered the morphology of the MDA-MB-453 cells from a mesenchymal to an epithelial phenotype. The results of western blot analysis revealed that BRACs increased the expression of the epithelial marker, E-cadherin, and decreased the expression of the mesenchymal markers, fibronectin and vimentin. These results indicate that the inhibitory effect of BRACs on EMT plays an important role in their anti-metastatic effects.

To delineate whether FAK signaling plays a significant role in the metastasis of MDA-MB-453 cells, the present study used adhesion, wound healing and Transwell assays to analyze the adhesion, migration, invasion and EMT status of cells pretreated with Y15. Y15 inhibited the phosphorylation of FAK by binding with the Y397 site. The Y397 site is an autophosphorylation site of FAK that provides a binding for cSrc to lead to downstream signaling by mediating the phosphorylation of FAK. A previous study demonstrated that Y15 inhibits the growth of breast cancers (34). The present study demosntrated that the adhesion, migration, invasion of the MDA-MB-453 cells and EMT were reduced by Y15. The aforementioned results indicated that BRACs exerted similar anti-metastatic effects to those of Y15, and taht FAK played a vital role in the anti-metastastic effects of BRACs.

In the present study, the expression and phosphorylation of FAK, cSrc and p130 ${ }^{\text {Cas }}$ were investigated. The total level of FAK, cSrc and p130 Cas was not affected, but the phosphorylation levels were reduced by BRACs and Y15. BRACs decreased the interaction between HER-2 and FAK, FAK and cSrc, cSrc and $\mathrm{p} 130^{\mathrm{Cas}}$, and between FAK and p130 ${ }^{\mathrm{Cas}}$. It also decreased the activation and transduction of FAK signaling. Taken together, this study suggests that BRACs suppress the metastasis of HER-2-positive breast cancer in vitro, and that the $\mathrm{cSrc} / \mathrm{FAK} / \mathrm{p} 130^{\mathrm{Cas}}$ pathway plays a vital role in this inhibitory effect (Fig. 6). It has been demonstrated anthocyanins from raspberry extract (RES) enhance the effects of radiotherapy and chemotherapy in the treatment of pancreatic cancer by controlling apoptosis and signaling pathways (35). Another study also indicated that RES enhanced the susceptibility of centchroman in breast cancer cells (36). These studies suggest anthocyanins combined with anticancer drugs may be used to enhance the anticancer effects. In conclusion, our results may probe usefule to future studies on BRACs using animals and humans. BRACs may have potential for use as supplementary treatments with anticancer drugs.

\section{Acknowledgements}

This study was supported by the National Natural Science Foundation of China (nos. 81273074 and 81573154), the Innovation Research Team Project of Sichuan Province Youth Science and Technology Foundation (no. 2014TD0021), and the Scientific Research Innovation Team Project of Sichuan Provincial University Foundation (no. 14TD00234). The authors would like to thank Mrs. Weiwei Zhang for assisting with the revision of the manuscript.

\section{References}

1. Friedenreich CM: Physical activity and breast cancer: Review of the epidemiologic evidence and biologic mechanisms. Recent Results Cancer Res 188: 125-139, 2011.

2. Zheng L, Zhou B, Meng X, Zhu W, Zuo A, Wang X, Jiang R and Yu S: A model of spontaneous mouse mammary tumor for human estrogen receptor- and progesterone receptor-negative breast cancer. Int J Oncol 45: 2241-2249, 2014.

3. Jemal A, Bray F, Center MM, Ferlay J, Ward E and Forman D: Global cancer statistics. CA Cancer J Clin 61: 69-90, 2011.

4. Mendoza G, Portillo A and Olmos-Soto J: Accurate breast cancer diagnosis through real-time PCR her-2 gene quantification using immunohistochemically-identified biopsies. Oncol Lett 5: 295-298, 2013.

5. Gutierrez C and Schiff R: HER2: Biology, detection, and clinical implications. Arch Pathol Lab Med 135: 55-62, 2011.

6. Slamon DJ, Godolphin W, Jones LA, Holt JA, Wong SG, Keith DE, Levin WJ, Stuart SG, Udove J, Ullrich A, et al: Studies of the HER-2/neu proto-oncogene in human breast and ovarian cancer. Science 244: 707-712, 1989.

7. Heerboth S, Housman G, Leary M, Longacre M, Byler S, Lapinska K, Willbanks A and Sarkar S: EMT and tumor metastasis. Clin Transl Med 4: 6, 2015.

8. Heffler M, Golubovskaya VM, Dunn KM and Cance W: Focal adhesion kinase autophosphorylation inhibition decreases colon cancer cell growth and enhances the efficacy of chemotherapy. Cancer Biol Ther 14: 761-772, 2013.

9. Golubovskaya VM: Targeting FAK in human cancer: From finding to first clinical trials. Front Biosci (Landmark Ed) 19: 687-706, 2014.

10. Vadlamudi RK, Sahin AA, Adam L, Wang RA and Kumar R: Heregulin and HER2 signaling selectively activates c-Src phosphorylation at tyrosine 215. FEBS Lett 543: 76-80, 2003.

11. Dolfi F, Garcia-Guzman M, Ojaniemi M, Nakamura H, Matsuda M and Vuori K: The adaptor protein Crk connects multiple cellular stimuli to the JNK signaling pathway. Proc Natl Acad Sci USA 95: 15394-15399, 1998.

12. Han C, Ding H, Casto B, Stoner GD and D'Ambrosio SM: Inhibition of the growth of premalignant and malignant human oral cell lines by extracts and components of black raspberries. Nutr Cancer 51: 207-217, 2005.

13. Fayyaz S, Aydin T, Cakir A, Gasparri ML, Panici PB and Farooqi AA: Oleuropein mediated targeting of signaling network in cancer. Curr Top Med Chem 16: 2477-2483, 2016.

14. Farooqi AA, Wang Z, Hasnain S, Attar R, Aslam A, Mansoor Q and Ismail $\mathrm{M}$ : Citrus fruits and their bioactive ingredients: Leading four horsemen from front. Asian Pac J Cancer Prev 16: 2575-2580, 2015.

15. Lin X, Farooqi AA and Ismail M: Recent progress in fungusderived bioactive agents for targeting of signaling machinery in cancer cells. Drug Des Devel Ther 9: 1797-1804, 2015.

16. Perk AA, Shatynska-Mytsyk I, Gerçek YC, Boztaş K, Yazgan M, Fayyaz S and Farooqi AA: Rutin mediated targeting of signaling machinery in cancer cells. Cancer Cell Int 14: 124, 2014.

17. Attar R, Tabassum S, Fayyaz S, Ahmad MS, Nogueira DR, Yaylim I, Timirci-Kahraman O, Kucukhuseyin O, Cacina C, Farooqi AA, et al: Natural products are the future of anticancer therapy: Preclinical and clinical advancements of Viscum album phytometabolites. Cell Mol Biol (Noisy-le-grand) 61: 62-68, 2015. 
18. Kong S, Kim DJ, Oh SK, Choi IS, Jeong HS and Lee J: Black rice bran as an ingredient in noodles: Chemical and functional evaluation. J Food Sci 77: C303-C307, 2012.

19. Sehitoglu MH, Farooqi AA, Qureshi MZ, Butt G and Aras A: Anthocyanins: Targeting of signaling networks in cancer cells. Asian Pac J Cancer Prev 15: 2379-2381, 2014.

20. Hui C, Bin Y, Xiaoping Y, Long Y, Chunye C, Mantian M and Wenhua L: Anticancer activities of an anthocyanin-rich extract from black rice against breast cancer cells in vitro and in vivo. Nutr Cancer 62: 1128-1136, 2010.

21. Luo LP, Han B, Yu XP, Chen XY, Zhou J, Chen W, Zhu YF, Peng XL, Zou Q and Li SY: Anti-metastasis activity of black rice anthocyanins against breast cancer: Analyses using an ErbB2 positive breast cancer cell line and tumoral xenograft model. Asian Pac J Cancer Prev 15: 6219-6225, 2014.

22. Chen XY, Zhou J, Luo LP, Han B, Li F, Chen JY, Zhu YF, Chen W and Yu XP: Black Rice Anthocyanins Suppress Metastasis of Breast Cancer Cells by Targeting RAS/RAF/MAPK Pathway. Biomed Res Int 2015: 414250, 2015.

23. Xu M, Bower KA, Wang S, Frank JA, Chen G, Ding M, Wang S Shi X, Ke Z and Luo J: Cyanidin-3-glucoside inhibits ethanolinduced invasion of breast cancer cells overexpressing ErbB2. Mol Cancer 9: 285, 2010.

24. Xu M, Bower KA, Chen G, Shi X, Dong Z, Ke Z and Luo J: Ethanol enhances the interaction of breast cancer cells overexpressing ErbB2 with fibronectin. Alcohol Clin Exp Res 34: 751-760, 2010

25. Hou DX: Potential mechanisms of cancer chemoprevention by anthocyanins. Curr Mol Med 3: 149-159, 2003.

26. Crozier A, Jaganath IB and Clifford MN: Dietary phenolics: Chemistry, bioavailability and effects on health. Nat Prod Rep 26: 1001-1043, 2009.

27. Scalbert A and Williamson G: Dietary intake and bioavailability of polyphenols. J Nutr 130 (Suppl 8): 2073S-2085S, 2000.

28. Manach C, Scalbert A, Morand C, Rémésy C and Jiménez L: Polyphenols: Food sources and bioavailability. Am J Clin Nutr 79: 727-747, 2004.
29. Kamiloglu S, Capanoglu E, Grootaert C and Van Camp J: Anthocyanin absorption and metabolism by human intestinal caco-2 cells - A review. Int J Mol Sci 16: 21555-21574, 2015.

30. Fang J: Bioavailability of anthocyanins. Drug Metab Rev 46: 508-520, 2014.

31. Chen PN, Kuo WH, Chiang CL, Chiou HL, Hsieh YS and Chu SC: Black rice anthocyanins inhibit cancer cells invasion via repressions of MMPs and u-PA expression. Chem Biol Interact 163: 218-229, 2006

32. Wang LS and Stoner GD: Anthocyanins and their role in cancer prevention. Cancer Lett 269: 281-290, 2008.

33. Chen JS, Huang XH, Wang Q, Huang JQ, Zhang LJ, Chen XL, Lei J and Cheng ZX: Sonic hedgehog signaling pathway induces cell migration and invasion through focal adhesion kinase/AKT signaling-mediated activation of matrix metalloproteinase (MMP)-2 and MMP-9 in liver cancer. Carcinogenesis 34: 10-19, 2013.

34. Golubovskaya VM, Nyberg C, Zheng M, Kweh F, Magis A, Ostrov D and Cance WG: A small molecule inhibitor, 1,2,4,5-benzenetetraamine tetrahydrochloride, targeting the y397 site of focal adhesion kinase decreases tumor growth. J Med Chem 51: 7405-7416, 2008.

35. Veeraraghavan J, Natarajan M, Lagisetty P, Awasthi V, Herman TS and Aravindan N: Impact of curcumin, raspberry extract, and neem leaf extract on rel protein-regulated cell death/radiosensitization in pancreatic cancer cells. Pancreas 40: 1107-1119, 2011.

36. Singh N, Zaidi D, Shyam H, Sharma R and Balapure AK: Polyphenols sensitization potentiates susceptibility of MCF-7 and MDA MB-231 cells to Centchroman. PLoS One 7: e37736, 2012.

This work is licensed under a Creative Commons Attribution-NonCommercial-NoDerivatives 4.0 International (CC BY-NC-ND 4.0) License. 